\title{
Utilization of Semantic Networks in the Teaching of Vocabulary
}

\author{
James D. Dunn \\ UNITAS at Teikyo University, Tokyo, Japan
}

\begin{abstract}
Cognitive neuroscience and psychology have shone light on new ways to help students learn, understand, and apply new vocabulary. The purpose of this paper is to present a theoretical method for the integration of semantic network utilization into the classroom. This paper will also introduce insights from Cognitive Linguistics as to how the brain best learns vocabulary. The method in this paper springs from the fields of psychology and neuroscience as well as inspiration from educators who are building new teaching styles. Semantic networks in the brain are the maps to understanding that hold the potential to make learning vocabulary more effective and meaningful. The purpose of the method detailed in this paper is to inspire other educators to incorporate cognitive linguistic insights into their classes as well as further the discourse on integrating this field into the teaching of English as a second or foreign language.
\end{abstract}

The method proposed in this paper is a theoretical model based on ideas such as Differentiated Instruction, emotionality in learning, cognitive linguistics, student schema, and semantic networks. While this method is not the result of direct collaboration with the educators mentioned within this paper, the author has implemented this method in his own classes at Teikyo University with success. Tomlinson's differentiated instruction (1999) weighs heavily in this method, as it influences the core concept of playing to the student's personal needs and interests during the course of an activity or lesson. This strategy combines well with Murphy's (2012) NeuroEFL strategies that emphasize the connections between emotionally engaged students and higher learning potentials. Murphy (2012) stated that students who are engaged in and emotionally connected to the material being presented have a higher potential for learning and retaining new information. There also exists the real possibility that the way students see the world is different from their peers. The way one understands the meaning and contextual use of a vocabulary term, as a collection of understanding and use, is known as schema (Littlemore, 2012). These differences give teachers the unique opportunity to combine student experiences and thoughts while they are emotionally engaged in vocabulary activities. Recurring activation of existing and new connections to the core concept helps to cement a new term into the mind (Jenkins, 2012a; Lin, 1997; Roediger \& McDermott, 1995). The theoretical method presented in this paper has been put into use by the author of this paper in university level classes with very good results, as measured by student satisfaction questionnaires and also against extensive reading vocabulary practice assessment scores. The author of this paper is currently collecting data in an action research to verify that this method provides benefits over other more traditionally accepted methods of vocabulary learning.

Language Education in Asia, 2013, 4(2), 175-182. http://dx.doi.org/10.5746/LEiA/13/V4/I2/A07/Dunn 


\section{History of Semantic Networks and Basic Assumptions}

The origin of the term network, as used to describe language memory and the brain, dates back to the 1970s (Leech, 1974; Lehrer, 1974) when the lexical structure of terms was being explored. The psycholinguist Aitchison (1987) researched how the brain organized its mental lexicon and coined two terms to describe how the brain stored vocabulary. The first, which will not be discussed at length in this paper, is the globule theory. When it is presented visually, the globule theory shows that word meanings are overlapping in meaning and contextual nature. This means that the location where one word's meaning ends cannot be precisely measured in relation to its neighboring, lexically-connected word. The theory also states that vocabulary terms can be traced back to semantic primitives which should hold similar meanings in all languages. The second is the cob-web theory, which evolved into what is known today as semantic networks (Aitchison, 1987). The cob-web theory shows the organizational nature of lexical terms stored in the brain by connecting related terms (e.g., hyponyms to hypernyms, or terms to their antonyms and indirect antonyms) to each other by drawing a network of interconnected lines and nodes (Jenkins, 2012a; Lin, 1997). This style of word mapping is one of the influencing ideas of the theoretical model proposed in this paper. One node, which is comprised of many interconnected terms, is connected to many others, which gives a fuller understanding of a term's meaning and contextual use. This deeper understanding of a vocabulary term helps language learners (LLs) to not only remember and recall vocabulary terms, but also to use them in correct contextual and anecdotal situations.

It can be argued that the more connections a new vocabulary term makes to a student's life when being learned, the stronger and more indelible the mark is on the LL's brain (Lin, 1997). By fleshing out a word's context, images, meanings, and schema relationships in class, educators help students master a new word more quickly and efficiently. Theoretical contributions from leaders in the cognitive linguistics field (Boers, Demecheleer, \& Eyckmans, 2004; Lazar, 1996; Littlemore, 2001) have indicated that applying this cognitive framework of interconnected meanings in thematic clusters aids learners of English as they progress towards mastery (Mirjalili, Jabbari, \& Rezai, 2012). New terms should also be readily connectable to experiences and situations in the students' lives that are relevant to them. These connectors, which are presented in an individualized manner by the teacher for each class as well as created by each student's individual experiences later in the method, create unique opportunities to put into practice cognitive linguistic insights in communicative activities in the classroom.

\section{Semantic Network Stimulation During Learning}

In the brain, memory comes from the connections among neurons that stay behind once a stimulus is experienced and processed in the lexicon. These connections are re-excited each time we need to recall a word or see a sight connected to the term. One can use the interconnected nature of semantic networks to the benefit of students. For example, when introducing the new lexical item obfuscate, a word which has been introduced in the author's classroom using this theoretical method, it is presented with connectors that the teacher deems both culturally and semantically appropriate exemplars (Figure 1). Teachers must also take into consideration the differentiated nature of exemplars by country or region. An example of this is using the connector ninja in an example sentence and the word map. Stimulation of connectors around a lexical item gives the new terms stronger connections, making them, in effect, more memorable. It has been shown that, with enough stimulation of a semantic network, the brain can be tricked into hearing a word that has not been presented to it (Roediger \& McDermott, 1995). Jenkins (2012b) demonstrated this at the Japan Association for Language Teaching (JALT) conference in 2012 by listing a string of terms which are connectors to an unsaid term. He showed that related terms were activated by spoken connectors and 
created a false memory (Roediger \& McDermott, 1995) in the brains of the listeners. These connections create the understanding of the term's meaning and context, which is housed in the student's schema (Littlemore, 2012). Once connected to lexical items or experiences in the student's mind, these semantically connected words, or connectors, are exploitable in that they reinforce the new term in the brain each time they are used or heard in the context of a lesson which introduces the aforementioned new term. By extension, by carefully choosing connectors to illustrate a new term, an educator can stimulate the target word or words in the network from the onset of the method. This selection of connectors ends up creating a stronger bond for the word; thus, it is more easily recalled for later use. This complements what many neuroscientists (Sousa, 2011) say about the plasticity of the brain.

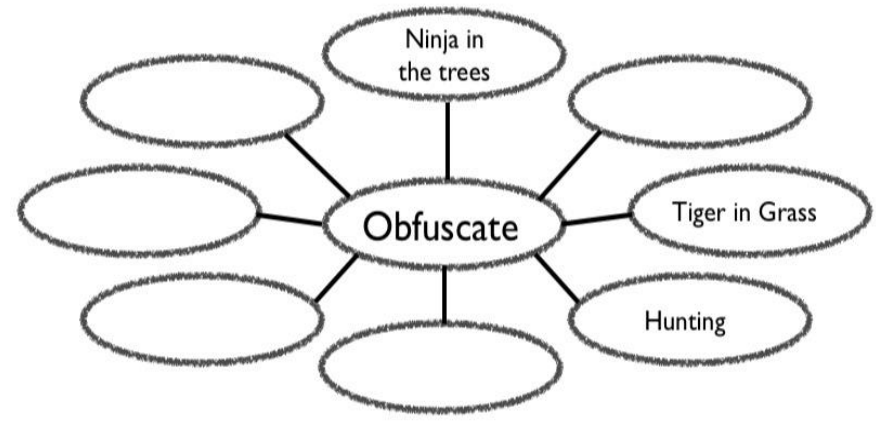

Figure 1. Example of a planned semantic network activity used in the author's class.

The more one uses the brain for an action or skill, the more lasting the memory that is created, due to reinforcement. The opposite is also true; if the knowledge of a word gained is not utilized, either in a direct or indirect manner, it can start to degrade over time and eventually be virtually inaccessible from one's working lexicon. This supports the popular saying "use it or lose it" as it applies to memories and language skills. Jenkins (2012a) showed that in a lexicon, there exist semantic nodes which can act as a focal point of understanding but at the same time are fuzzy in that it is difficult to determine where one concept ends and another one begins. This example lends credibility to the globule theory put forth by Aitchison (1987) that lexical entries are connected and overlapping, thus creating the fuzzy nature of the nodes. While educators, through experience and reflective practice, can improve their sense of what works in class, neuroscience and cognitive psychology are making inroads into new best practices that are still being developed. Presenting terms with associated, thematically-connected items helps to cement new words in the brain's lexical network and give appropriate contexts for use and understanding (Lin, 1997; Mirjalili, Jabbari, \& Rezai, 2012). Educators must also take into consideration the various differences between students' perceptions of terms, or construals (Littlemore, 2012), and use those differences to help in the learning process.

\section{Students' Individual Semantic Networks}

Educators know that not all students are the same and, as such, cannot be expected to learn exactly the same way in all situations. Murphy (2012) has stated that human beings' memories are the sum of their connections. In essence, one's reality is shaped by these connections in the brain. One's experiences make up how those connections are formed and explain why one understands terms or ideas differently from others. Littlemore (2001) stated that these differences of understanding are from an individual's schema. Differences in learning styles will also color one's reality and how new terms are coded and understood in the brain. Differentiated instruction implies that it is important to take differences of understanding and experience, as well as socio-economic status, culture, and other factors into consideration when attempting to teach new concepts to students (Tomlinson, 1999). By utilizing students' 
experiences in life and differences in their schema, as well as connecting well-known or culturally significant images that reinforce vocabulary meaning in students' lives, educators can make lessons more emotionally charged and meaningful for their students. Taking students into consideration when planning activities also provides opportunities for practice that might otherwise not have been presented before. By knowing, for example, that students are interested in a new video game, the teacher can exploit this knowledge by including information, terms, or even the name of the game into a connector example to create engagement in the lesson.

Languages, and the associated terms therein, require context to have meaning; thus, using a student's past experiences and original ideas in the learning process makes the learning experience emotionally charged. Providing students with opportunities to expand their semantic networks as they see fit allows them to further incorporate new words into their existing semantic nodes that surround the new term (Figure 2). Students should also have the chance to express why they have added a term as a connector, thus giving them opportunities to use and reinforce the new term and continually activate the new lexical item's semantic node. Utilizing student-created connectors gives new terms greater depth and breadth of meaning to students than they could get from using a dictionary (Anderson, 2011). This also allows for the content to be emotionally charged, as the students use original content and ideas to connect the new term to their lives while adding depth and breadth to its meaning. Learning that is emotionally charged is a powerful spur for retention in the brain and keeps students motivated and interested in the subject at hand (Murphy, 2012).

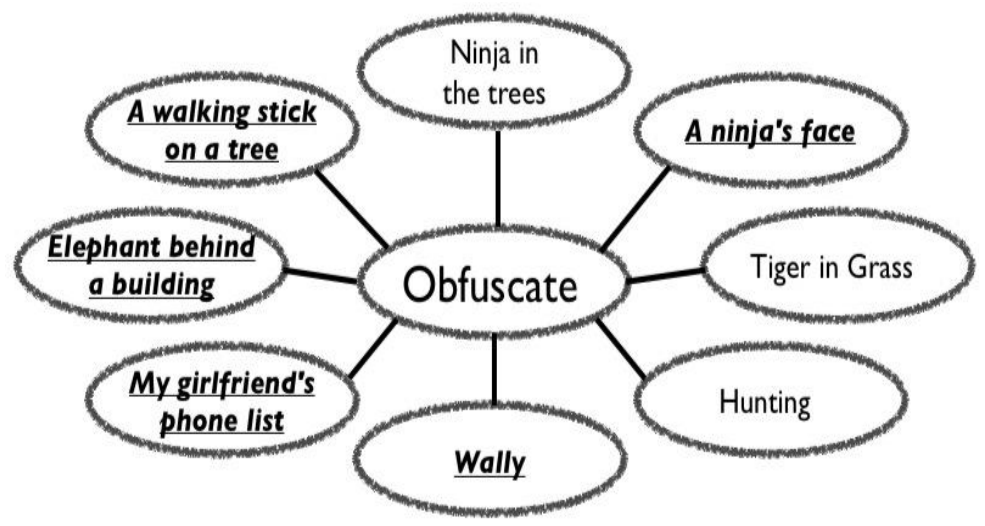

Figure 2. Example of semantic network with student ideas added. Underlined words denote actual student ideas as presented in the author's class.

The lexical construal differences among students can also be utilized in a communicative manner during the class to further enhance the understanding, context, and significance of new terms. This sharing of emotionally connected examples is also important in making more connections in semantic networks by exploring schematic differences among students. This provides excellent openings for students to express themselves in a communicative manner with a controlled group of new vocabulary terms while simultaneously reinforcing new terms by activating their semantic networks as they discuss their connectors.

\section{Theoretical Method for Utilizing Semantic Networks}

There are many methods that are available for educators to employ to teach vocabulary, but there are still achievement gaps in learning among students. No two students are the same and, as the understanding of specific cognitive and neurological mechanisms for learning improves, educators are constantly developing new methods to instruct students. The purpose of the 
following sections is to present a theoretical method, with associated activities, that has been both interesting for students and effective for vocabulary learning in the author's classes at Teikyo University. The following method utilizes semantic networks either directly or indirectly to teach, reinforce, and encourage the use of new terms. Two teaching styles, top-down, in part one of the method, and bottom-up, in part three, are simultaneously utilized for students to gain a fuller understanding of a new term. The purpose of using both styles is to create an emotionally-charged learning environment; this is referred to as the sandwich teaching method by Murphy (2012). The sandwich method leads to more motivation, better understanding, and an emotional learning atmosphere because teachers are giving information to and receiving information from students.

\section{Part 1: Contextual Clues}

First in this theoretical method of vocabulary instruction is utilizing contextual clues in sample sentences. Using context clues supports the learning done with semantic network practice and provides a valuable skill that students can use beyond the classroom. When students are presented with a new word in the context of a sentence that has been specifically designed by the teacher to show its meaning and context, powerful implicit learning occurs. Terms are also introduced with visual, auditory, or other stimuli to aid contextual comprehension and determination of their meaning (Figure 3). Students are given the opportunity to read the list of sample terms and use the information provided in contextually correct sample sentences to find the meaning of a word. While a term can be presented in different contexts, a few sentences which have been carefully crafted by the teacher should suffice to start a student on the road to inquiry-based learning.

\section{What does it mean to me? How about you?}

(Expanding meanings of words through communication)

(Word: Obfuscate)

\section{FIRST}

Read the sentences and decide what type of word it is and the meaning from the context of the sentence. Context is kind of like the 空気 of a sentence. It tells us a lot.

VOCABULARY ONE

The tiger was obfuscated by the tall grass as it hunted the zebra.

A ninja obfuscated himself in the trees, waiting for the shogun to pass under.

What kind of word is "obfuscate"? Noun Verb Adjective

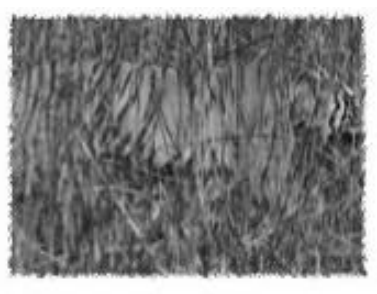

Figure 3. Example of contextual clues used in class to introduce the target word obfuscate.

Students would then be given a chance to explain the part of speech it is and the meaning as they see it. Providing opportunities to ask follow-up questions about peer interpretations encourages curiosity and allows gains in semantic knowledge through schema sharing (Figure 4). The teacher must also be aware of what is going on to ensure that any misunderstandings that occur, e.g., when a student creates an incorrect or incomplete meaning from the context of a sentence, are corrected. 
What does it mean?

Share your ideas with your partner. Write his or her ideas below.

Figure 4. Example of meaning sharing for the target word obfuscate.

As previously mentioned, no student will have exactly the same definition, contextual knowledge, or vision of a new term. Utilizing a student's individual understanding of the world plays directly into differentiated instruction (Tomlinson, 1999) and emotional learning (Murphy, 2012), both of which are shown to improve learning by creating a positive learning environment that employs the student's own ideas and opinions in the learning process.

\section{Part 2: Schema Mapping}

Once a new term has been introduced to the class, students are given a word map with the new term in the center presented along with iconic examples carefully chosen for cultural and age appropriateness to get the students started (Figure 5). Semantic maps allow students to map out word meanings on a segmented map which connects a target word to multiple, semantically connected terms (Suhor \& Little, 1988). In this part of the method, teachers make use of these types of semantic maps in a thematic fashion, expanding out from only semantically connected words. Teachers do this by having students write words for people, creatures, emotions, and places for their connectors to the target word. Emotionally charged connectors such as these positively influence learning (Murphy, 2012). Students continue by filling in their own connectors to the vocabulary term in the schema map. During this time, the teacher monitors the students' progress and helps to clarify meanings, correct flaws in their understanding, or correct the use of inappropriate connectors. Students are encouraged to write different types of connectors that, in their schema, connect to the target word. This activity allows students to follow their own mental images and understanding of a term and connect it to their lives, making learning a more implicit experience. This part of the method is largely dependent on the students' life experiences and willingness to share with their peers.

\section{NOW}

Write in five connected words that you can think of for "obfuscate." (example: ninja, hunting, tiger in grass)

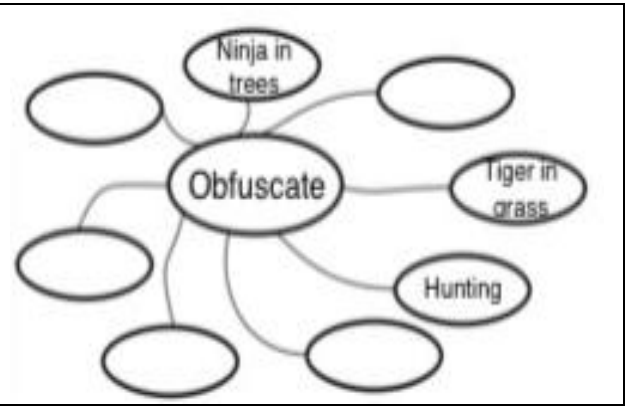

Figure 5. Example of schema mapping for the target word obfuscate.

\section{Part 3: Sharing Schema and Increasing Depth and Breadth of the New Word}

Once the semantic map of a term has been completed, putting the students into pairs or groups allows them to discuss their maps with each other and then explain why they have added their connectors to the map (Figure 6). The teacher needs to encourage the students' curiosity at first by supporting follow-up questions with praise and showing interest in their explanations as to why they have chosen a connector. The teacher can even encourage short debates among groups as to if a connector belongs in the semantic network. Once sufficient time has been given to discuss the meaning, as understood by their peers, and practice the new term, students use a prepared questionnaire to further practice using the term in a more directed communicative manner. The questionnaire also provides students with opportunities to write their own questions to reinforce the new term and make the learning process more emotionally 
charged.

\section{FINALLY}

Talk to a person around you and compare the connected words that he / she put in the word tree. Add two of your partner's words below and ask why the words were added. Write the reason for the connection next to the word. Make sure to ask follow-up questions to find out more about the reasons behind the connections.

Word 1 Why added:

Word 2 Why added:

Figure 6. Example of a questionnaire for schema sharing for the target word obfuscate.

\section{Conclusion and Further Considerations}

Utilizing semantic links to reinforce as well as broaden the understanding of a new vocabulary term, while taking into consideration the differences among students' schema and cultural identities, is a method of instruction that has yet to be proven through quantitative research. The research currently available on lexical structures, differentiated instruction, utilization of multiple teaching styles, and semantic networks as ways to allow students to become more interested in a subject and learn in an effective manner is copious. Combining these different facets of pedagogy into a method creates an atmosphere of communication, learning, and interest. This theoretical method also creates an emotionally charged learning environment by using student ideas and differences in their schema, experiences, and opinions to further enhance the effectiveness of the teacher's lessons. By learning vocabulary with both depth and breadth of knowledge, students are able to utilize multiple paths to express themselves and convey their thoughts, opinions, and ideas to others. Gains in communicative ability, as well as standardized test scores, are believed to be products of having a contextually correct and deep lexicon. It must be noted that further research into the gains and their longevity is needed to push this method onto more solid ground as it is as yet unproven. Quantitative measures of the method's effectiveness must be obtained to prove or disprove its worth as a methodology. The author of this paper is currently engaged in an action research to help prove the effectiveness of this method as compared to other vocabulary teaching methods.

\section{Author Note}

James D. Dunn, UNITAS, Teikyo University, Hachioji City, Tokyo, Japan.

Correspondence concerning this article should be addressed to James Dunn, Kurihira 1-7-7, Room 602, Asao-ku, Kawasaki-shi, Kanagawa-ken 215-0031, Japan. E-mail: James.D.Dunn@outlook.com 


\section{References}

Aitchison, J. (1987). Words in the mind: An introduction to the mental lexicon. Cambridge, MA: Basil Blackwell.

Anderson, O. (2011). Brain, mind, and the organization of knowledge for effective recall and application. Learning Landscapes, 5(1), 45-62.

Boers, F., Demecheleer, M., \& Eyckmans, J. (2004). Cross-cultural variation as a variable in comprehending and remembering figurative idioms. European Journal of English Studies, 8(3), 375-388. http://dx.doi.org/10.1080/1382557042000277449

Jenkins, A. (2012a). Neuroscience, psychology, and the teaching of vocabulary. Center for Student Education Resource, 65-68. Retrieved from: http://www.sist.ac.jp/lib/Ejournal/bulletin-PDF/kiyou20/kiyou20_9_Jenkins.pdf

Jenkins, A. (2012b, October). Neuroscience, psychology, and the teaching of vocabulary. Paper presented at the meeting of the Japan Association for Language Teaching, Hamamatsu, Japan.

Lazar, G. (1996). Using figurative language to expand students' vocabulary. ELT Journal, 5011), 43-51. http://dx.doi.org/10.1093/elt/50.1.43

Leech, G. (1974). Semantics. Harmondsworth, England: Penguin.

Lehrer, A. (1974). Semantic fields and lexical structure. New York, NY: American Elsevier.

Lin, C. (1997). Semantic network for vocabulary teaching. Journal of Taiwan Normal University: Humanities \& Social Science, 42, 43-54. Available at http://140.122.100.145/ntnuj/j42/hs42-4.pdf

Littlemore, J. (2001). An empirical study of the relationship between cognitive style and the use of communication strategy. Applied Linguistics, 22(2), 241-265. http://dx.doi.org/10.1093/applin/22.2.241

Littlemore, J. (2012, October). Cognitive linguistics and second language learning. Paper presented at the meeting of the Japan Association for Language Teaching, Hamamatsu, Japan.

Mirjalili, F., Jabbari, A. A., \& Rezai, M. J. (2012). The effect of semantic and thematic clustering of words on Iranians vocabulary learning. American International Journal of Contemporary Research, 2(2), 214-222. Available at http://www.aijcrnet.com/journals/Vol_2_No_2_February_2012/26.pdf

Murphy, R. (2012, October). Neuroscience \& EFL: Super-dynamic teaching. Paper presented at the meeting of the Japan Association for Language Teaching, Hamamatsu, Japan.

Roediger, H. L., \& McDermott, K. B. (1995). Creating false memories: Remembering words not presented in lists. Journal of Experimental Psychology: Learning, Memory, and Cognition, 21(4), 803-814. http://dx.doi.org/10.1037/0278-7393.21.4.803

Sousa, D. (2011). Mind, brain, and education: The impact of educational neuroscience on the science of teaching. Learning Landscapes, 5(1), 37-44.

Suhor, C., \& Little, D. (1988). Visual literacy and print literacy: Theoretical considerations and points of contact. Reading Psychology, 9(4), 469-481. http://dx.doi.org/10.1080/0270271880090412

Tomlinson, C. (1999). The differentiated classroom: Responding to the needs of all learners. Alexandria, VA: Association for Supervision and Curriculum Development. 